\title{
Endoscopic Ultrasound-Guided Vascular Therapy: The Present and the Future
}

\author{
Philip S.J. Hall, Christopher Teshima, Gary R. May and Jeffrey D. Mosko \\ The Center for Therapeutic Endoscopy and Endoscopic Oncology, St. Michael's Hospital, University of Toronto, ON, Canada
}

Endoscopic ultrasound (EUS) offers access to many intra-abdominal vessels that until now have only been accessible to the surgeon and interventional radiologist. In addition to assisting with diagnostics, this unique access offers the potential for therapeutic intervention for a host of indications. To date, this has had the most clinical impact in the treatment of gastroesophageal varices, with EUS-guided coil and glue application growing in use worldwide. Although randomised controlled trial data is lacking, we discuss the growing body of literature behind EUS-guided therapy in the management of varices. EUS has also been used in specialized centres to assist in nonvariceal gastrointestinal bleeding. The treatment of bleeding from Dieulafoy lesions, tumours and pancreatic pseudoaneurysms has all been described. The potential applications of EUS have also extended to the placement of portal vein stents and porto-systemic shunts in animal models. As medicine continues to move to increasingly less invasive interventions, EUS-guided therapies offer substantial promise for the safe and effective delivery of targeted treatment for a widening array of vascular disorders. Clin Endosc 2017;50:138-142

Key Words: Endosonography; Esophageal and gastric varices; Portal pressure

\section{INTRODUCTION}

In recent years, the role of endoscopic ultrasound (EUS) has expanded rapidly into the therapeutic arena. From its origins in diagnostics and tissue acquisition, EUS-guided therapy now offers options in the management of pancreatic fluid collections, difficult pancreaticobiliary access and in the treatment of gastroesophageal varices.

As mentioned, EUS offers unique access to abdominal arterial and venous vasculature that until now has only been accessible to surgeons and/or interventional radiologists. This has had the most clinical impact on the treatment of gastroesophageal varices, where EUS may play a role both in the

Received: March 19, 2017 Accepted: March 23, 2017

Correspondence: Jeffrey D. Mosko

The Center for Therapeutic Endoscopy and Endoscopic Oncology, St. Michael's Hospital, University of Toronto, 16-034 Cardinal Carter Wing, Toronto, ON M5B 1W8, Canada

Tel: +1-416-864-5684, Fax: +1-416-864-5882, E-mail: moskoj@smh.ca

(cc) This is an Open Access article distributed under the terms of the Creative Commons Attribution Non-Commercial License (http://creativecommons.org/ licenses/by-nc/3.0) which permits unrestricted non-commercial use, distribution, and reproduction in any medium, provided the original work is properly cited. acute and elective management and can deliver therapy in the form of glue injection, endovascular coil placement or a combination of the two.

While only currently described in animal studies and case series respectively, EUS can facilitate portal pressure measurement and assist in the management of refractory gastrointestinal bleeding.

This article aims to discuss the body of evidence pertaining to the EUS-guided management of varices as well as to explore available evidence regarding other vascular therapies that have yet to be incorporated into or recommended for everyday clinical practice.

\section{ESOPHAGEAL VARICES}

Direct endoscopic therapy for both elective and emergent esophageal variceal bleeding is well established and effective. Forward viewing endoscopy outperforms EUS at both the detection and grading of esophageal varices, however EUS has been shown to be efficacious in assessing potential 'feeding' veins. ${ }^{1}$ The size of such peri-esophageal veins appears to cor- 
relate with bleeding risk. ${ }^{2}$ Several studies have also confirmed that the ongoing presence of these veins after endoscopic treatment is associated with an increased risk of recurrence. ${ }^{3,4}$

Peri-esophageal veins offer a potential therapeutic target for EUS-guided therapy. Lahoti et al. treated 5 patients with the direct injection of sclerosant into perforating esophageal vessels with obliteration of varices in an average of 2.2 treatments. ${ }^{5}$ No recurrence of bleeding was reported after 15 months of follow up. De Paulo et al. conducted a randomised, controlled trial comparing standard endoscopic sclerotherapy with EUS-guided sclerotherapy to treat collateral vessels. ${ }^{6}$ The authors showed a trend toward delayed recurrence of varices following EUS-guided therapy, however their results did not reach statistical significance. Neither the amount of sclerosant nor sessions required to achieve eradication were significantly different.

EUS-guided treatment of perforating vessels in cases of esophageal variceal bleeding is an attractive option. Further trials demonstrating superiority over standard endoscopic therapy, potentially using cyanoacrylate (CYA) glue injection in place of sclerosant, would be required before it is adopted into any therapeutic algorithm.

\section{GASTRIC VARICES}

The commonest vascular application of EUS is in the diagnosis and treatment of gastric varices. Gastric varices account for $10 \%-30 \%$ of variceal bleeding episodes and are associated with high morbidity and mortality. The bleeding in these patients tends to be severe and between 35\%-90\% will re-bleed after spontaneous hemostasis. ${ }^{8}$

Injection with CYA glue has become the standard of care for both acute bleeding and secondary prophylaxis. CYA injection has been reported to achieve hemostasis in 80\%-90\% of bleeding cases, with low rates of re-bleeding. ${ }^{9}$

Unlike with esophageal varices, the use of EUS offers a clear advantage in the diagnosis of gastric varices. ${ }^{10}$ The use of colour Doppler can reliably differentiate gastric varices from other fundal lesions such as gastrointestinal stromal tumours and thickened gastric folds. EUS has also been used to evaluate the response to gluing. Lee et al. retrospectively compared two groups of patients who received endoscopic (forward viewing) glue treatment for bleeding gastric varices. ${ }^{11}$ The first group of 47 patients had repeat treatment on demand, whilst the second group of 54 patients had biweekly EUS and glue injection until obliteration of flow was confirmed on EUS. Although early re-bleeding rates were similar, late re-bleeding rates ( $>48$ hours) were significantly lower in the EUS group (19\% vs. $45 \%, p=0.005)$.

\section{EUS-guided glue injection}

EUS-guided glue therapy offers a range of potential advantages over standard glue injection. In addition to the accurate delivery of glue into the target varix, EUS enables the operator to confirm obliteration of the varix by using colour Doppler. EUS also allows for identification and subsequent glue delivery into the perforating or feeding vessels, theoretically decreasing the amount of glue required and minimising the risk of embolization. Romero-Castro et al. published a small proof of concept case series describing the use of EUS to target perforating vessels for glue injection using a $22 \mathrm{G}$ needle. ${ }^{12}$ They used Doppler to confirm obliteration of the afferent feeding vessels after injection of a CYA/lipiodol solution. The $5 \mathrm{pa}-$ tients in the study had no further bleeding or complications in a short follow up period. However, the process of determining the exact feeding vessel was described as difficult and time consuming. Their technique necessitated the injection of contrast medium prior to glue to ensure that the afferent feeding vessel was correctly selected. ${ }^{13}$

\section{EUS-guided coil embolization}

The second EUS-guided therapeutic option for treating gastric varices involves the placement of micro-coils into the varices leading to obliteration. These metal coils are covered with synthetic fibres which promote clot aggregation and can provide a scaffold for glue adherence. They are available in a variety of sizes that can be matched to the diameter of the target varices. Once the varix has been punctured using a standard EUS access needle, the coils are advanced through the needle and into the target varix using the needle's stylet as a 'pusher'. Most coils can be advanced through a $19 \mathrm{G}$ access needle (up to 0.035-inch coils) whilst smaller 0.018inch coils are available for use with $22 \mathrm{G}$ needles. Since this technique was first described in isolated case series, its use has increased in recent years both as monotherapy and in combination with CYA. ${ }^{14,15}$

A multicentre cohort study compared EUS-guided CYA application with EUS-guided coiling. ${ }^{16}$ Of the 30 patients enrolled, 19 underwent CYA injection while 11 received EUS-guided coiling. After 6 months of follow up, there was no significant difference in the rate of obliteration of gastric varices (95\% of the CYA group versus $91 \%$ of the coil group). A higher number of sessions were required to achieve obliteration with CYA, but due to the sample size, this did not reach statistical significance. The authors did show that $82 \%$ of the EUS-guided coiling group had complete obliteration after only one session. The risk of complications from CYA injection was significantly higher than with EUS-guided coiling (58\% vs. $9 \%$, respectively). However, this included 9 patients found to have asymptomatic pulmonary emboli 
from glue injection, of unlikely clinical significance. There were no deaths and no bleeding complications in the study.

\section{EUS-guided coil and glue combination}

In 2011, Binmoeller et al. reported on the use of a combination of EUS-guided coil deployment with glue injection. ${ }^{17}$ They postulated that the use of the coil would give the CYA a scaffold on which to form, minimising the amount of glue required and the risk of embolization. ${ }^{13}$ They evaluated combination therapy in 30 patients with gastric varices $>1 \mathrm{~cm}$ that were not good candidates for transjugular intrahepatic portosystemic shunt (TIPSS). Hemostasis was achieved in all actively bleeding patients. One session of treatment was enough to achieve obliteration of the target gastric varix in $96 \%$ of patients. The amount of glue required was $1.4 \mathrm{~mL}$ per varix, which was $1 \mathrm{~mL}$ less, on average, than previous studies of glue alone from the same institution. Treatment of gastric varices in this study was performed using a trans-esophageal approach. EUS offers excellent visualization of the gastric fundus through the distal esophagus, particularly when the stomach is filled with water. This approach is attractive as treatment is not limited by gastric contents or blood/blood clots overlying the target varices which can make standard endoscopic therapy in a retroflexed position quite difficult.

A recently published study by Bhat et al. documented long term follow up (mean, 436 days) in 152 patients treated with a combination of EUS-guided CYA injection and coiling. ${ }^{18}$ Their data revealed that the initial procedure attempt was successful in achieving hemostasis in 151 out of 152 cases, with a $7 \%$ rate of post procedure complications. Of these, the most serious was a single symptomatic pulmonary embolism in which the patient recovered fully with standard therapy. 100 patients underwent follow-up EUS, of whom $93 \%$ had confirmed obliteration of the target varix. Recurrent bleeding attributable to gastric varices in their patient population was $8 \%$.

A subgroup analysis from this study looked at 40 patients with high-risk gastric varices and no history of bleeding episodes. ${ }^{18}$ They were all treated with a combination of EUS-guided coil and CYA application. After a mean follow up of 449 days, only 2 bleeding episodes were reported. In both patients, bleeding occurred from new varices and were both successfully treated endoscopically. In those patients with documented EUS follow up, obliteration of the target varix was successful in $96 \%$ of patients. This data supports the consideration of primary prophylaxis of gastric varices with high-risk features by using a combination of EUS-guided coil embolization and CYA glue injection.

Given the high mortality rate from gastric variceal bleeding coupled with a lack of alternative endoscopic rescue therapies, the above data strongly supports the efficacy of EUS-guided glue injection or the combination of coil and glue therapy in patients with active or recent gastric variceal bleeding.

\section{Primary prophylaxis of gastric varices}

Should gastric varices be treated prophylactically? A randomised study by Mishra et al. treated patients with CYA, propranolol or no therapy. ${ }^{19}$ They included 89 patients with type 2 gastroesophageal varices (GOV-2) or type 1 isolated gastric varices (IGV-1) that were greater than $10 \mathrm{~mm}$ in diameter. Over 26 months, they found that bleeding episodes occurred in $10 \%$ of the CYA group, $38 \%$ of the propranolol group and $53 \%$ of the conservative management group. This study has subsequently been criticised for its small size and high failure rate with propranolol when compared with similar studies, ${ }^{20}$ but nevertheless suggests a benefit from primary prophylaxis. Two recent guidelines from the American Association for the Study of Liver Disease (AASLD, 2017) and the British Society of Gastroenterology (BSG, 2015) quote the study by Mishra et al., ${ }^{19}$ but note its limitations and thus do not recommend the use of CYA for primary prophylaxis in gastric varices outside of clinical trials. ${ }^{20,21}$ Both groups recommend non-selective beta blockade in these patients.

The study by Bhat et al. provides new information regarding the benefit of primary prophylaxis that was not available at the time of the creation of these guidelines. ${ }^{18}$ The low number of bleeding episodes among patients who received primary prophylaxis is notable ( 2 out of 40 patients, 449 days follow up). Confirmation of the benefit of using EUS-guided glue injection, coil embolization or a combination thereof in primary prophylaxis of gastric varices is needed in the form of a large randomised controlled trial.

\section{ECTOPIC VARICEAL THERAPY}

EUS-guided therapy has been described in case reports for variceal bleeding at other anatomical sites. So et al. described the use of an EUS-guided coil to halt massive duodenal variceal bleeding. ${ }^{22}$ The use of CYA glue in this scenario has also been described. Rectal varices are common but have a smaller risk of bleeding than their gastroduodenal counterparts. The uses of both EUS-guided CYA injection and combination therapy with EUS-guided coil/CYA for rectal varices have been described. ${ }^{23,24}$ EUS has also been used to direct glue into parastomal varices. ${ }^{25}$ While there is currently insufficient evidence to recommend EUS-guided treatment as first line in these cases, it has emerged as a viable option for rescue therapy. 


\section{PORTAL PRESSURE}

Current rescue therapy for refractory gastroesophageal variceal bleeding is the insertion of a TIPSS. EUS offers unique access to intra-abdominal vasculature including the hepatic and portal veins. The creation of an EUS-guided intrahepatic portosystemic shunt was first described in $2009 .{ }^{26}$ Schulman et al. have recently elaborated on this with a case series of EUS-guided intrahepatic portosystemic shunt formation in 5 porcine models with concurrent portal pressure monitoring. ${ }^{27}$ They describe accessing the hepatic vein or inferior vena cava (IVC) using a $19 \mathrm{G}$ needle and advancing the needle into the portal vein, taking pressure measurements at each position. They then deployed a lumen-apposing metal stent over a guidewire using fluoroscopic guidance to establish the shunt. They showed excellent technical success and an average procedure time of 43 minutes. In addition, Park et al. described the placement of transhepatic portal vein stents in 6 pigs, using the left intrahepatic portal vein as a point of access. $^{28}$

While the above therapies have only being trialled in animal models to date, they provide an exciting glimpse into the future potential for EUS-guided interventions. Endoscopists will have the capacity to choose from multiple EUS-guided therapies in the acute and rescue settings. Whether these therapies will be shown to provide benefit over current approaches provided by interventional radiology remains to be seen.

\section{NON-VARICEAL GASTROINTESTINAL BLEEDING}

EUS provides a potential alternative approach to patients with refractory gastrointestinal bleeding who have failed standard treatment with clips, epinephrine and electrosurgical coagulation. At present, EUS-guided therapy for these patients has only been described in case reports and case series. Levy et al. described therapy for a Dieulafoy lesion, gastrointestinal stromal tumor (GIST), duodenal ulcer and hemosuccus pancreaticus in patients who had failed standard endoscopic and interventional radiological intervention. ${ }^{29}$ They described the identification of feeder vessels and then injection of CYA or $99 \%$ alcohol via EUS using a $22 \mathrm{G}$ needle. They then used Doppler to confirm the absence of blood flow after therapy and reported a 100\% success rate with no re-bleeding at 12 months. Gonzalez et al. have reported on the treatment of Dieulafoy lesions, a bleeding pancreatic tumor, a pancreatic pseudoaneurysm, and an intracystic splenic artery pseudoaneursym. ${ }^{30,31}$ Thrombin injection via
EUS for pancreatic pseudoaneurysm bleeding has also been described. ${ }^{32}$ One of the largest series of EUS-guided interventions was reported by Law et al. ${ }^{33}$ They included 17 cases of refractory bleeding where alternative methods of control were not suitable. Combinations of glue, coils, alcohol and epinephrine injection were used to target bleeding lesions using a linear echoendoscope. They reported $88 \%$ success over a 12-month follow-up to prevent recurrent bleeding.

Whilst such reports offer an exciting glimpse into future therapies, there remains a lack of data supporting their use compared to standard therapies currently provided by interventional radiologists (conventional angiography with embolization). As such, EUS should only be considered an option in expert centres when all other treatment modalities have been unsuccessful.

\section{CONCLUSIONS}

The therapeutic applications of EUS continue to expand at a rapid rate. There is now literature, albeit few randomised, controlled trials, supporting the use of EUS-guided therapy (coil plus CYA) for the treatment of gastric varices. Case reports and case series provide insight into the potential of this modality for the treatment of refractory non-variceal bleeding. EUS-guided portal pressure measurement and portosystemic stenting has been performed in animal studies and may allow for improved outcomes with fewer interventions. As medicine continues to move to increasingly less invasive interventions, EUS-guided therapies offer substantial promise for the safe and effective delivery of targeted treatment options for a widening array of vascular disorders.

\section{Conflicts of Interest}

The authors have no financial conflicts of interest.

\section{REFERENCES}

1. Burtin P, Calès P, Oberti F, et al. Endoscopic ultrasonographic signs of portal hypertension in cirrhosis. Gastrointest Endosc 1996;44:257-261.

2. Faigel DO, Rosen HR, Sasaki A, Flora K, Benner K. EUS in cirrhotic patients with and without prior variceal hemorrhage in comparison with noncirrhotic control subjects. Gastrointest Endosc 2000;52:455-462.

3. Irisawa A, Saito A, Obara K, et al. Endoscopic recurrence of esophageal varices is associated with the specific EUS abnormalities: severe periesophageal collateral veins and large perforating veins. Gastrointest Endosc 2001;53:77-84.

4. Sato T, Yamazaki K, Toyota J, Karino Y, Ohmura T, Akaike J. Endoscopic ultrasonographic evaluation of hemodynamics related to variceal relapse in esophageal variceal patients. Hepatol Res 2009;39:126-133.

5. Lahoti S, Catalano MF, Alcocer E, Hogan WJ, Geenen JE. Obliteration of esophageal varices using EUS-guided sclerotherapy with color Doppler. Gastrointest Endosc 2000;51:331-333.

6. de Paulo GA, Ardengh JC, Nakao FS, Ferrari AP. Treatment of esoph- 
ageal varices: a randomized controlled trial comparing endoscopic sclerotherapy and EUS-guided sclerotherapy of esophageal collateral veins. Gastrointest Endosc 2006;63:396-402; quiz 463.

7. Sarin SK, Lahoti D, Saxena SP, Murthy NS, Makwana UK. Prevalence, classification and natural history of gastric varices: a longterm follow-up study in 568 portal hypertension patients. Hepatology 1992;16:1343-1349.

8. Wani ZA, Bhat RA, Bhadoria AS, Maiwall R, Choudhury A. Gastric varices: classification, endoscopic and ultrasonographic management. J Res Med Sci 2015;20:1200-1207.

9. Al-Hillawi L, Wong T, Tritto G, Berry PA. Pitfalls in histoacryl glue injection therapy for oesophageal, gastric and ectopic varices: a review. World J Gastrointest Surg 2016;8:729-734.

10. Choudhuri G, Dhiman RK, Agarwal DK. Endosonographic evaluation of the venous anatomy around the gastro-esophageal junction in patients with portal hypertension. Hepatogastroenterology 1996;43:12501255.

11. Lee YT, Chan FK, Ng EK, et al. EUS-guided injection of cyanoacrylate for bleeding gastric varices. Gastrointest Endosc 2000;52:168-174.

12. Romero-Castro R, Pellicer-Bautista FJ, Jimenez-Saenz M, et al. EUS-guided injection of cyanoacrylate in perforating feeding veins in gastric varices: results in 5 cases. Gastrointest Endosc 2007;66:402-407.

13. Weilert F, Binmoeller KF. New endoscopic technologies and procedural advances for endoscopic hemostasis. Clin Gastroenterol Hepatol 2016;14:1234-1244

14. Levy MJ, Wong Kee Song LM, Kendrick ML, Misra S, Gostout CJ. EUS-guided coil embolization for refractory ectopic variceal bleeding (with videos). Gastrointest Endosc 2008;67:572-574.

15. Romero-Castro R, Pellicer-Bautista F, Giovannini M, et al. Endoscopic ultrasound (EUS)-guided coil embolization therapy in gastric varices. Endoscopy 2010;42 Suppl 2:E35-E36.

16. Romero-Castro R, Ellrichmann M, Ortiz-Moyano C, et al. EUS-guided coil versus cyanoacrylate therapy for the treatment of gastric varices: a multicenter study (with videos). Gastrointest Endosc 2013;78:711-721.

17. Binmoeller KF, Weilert F, Shah JN, Kim J. EUS-guided transesophageal treatment of gastric fundal varices with combined coiling and cyanoacrylate glue injection (with videos). Gastrointest Endosc 2011;74:10191025.

18. Bhat YM, Weilert F, Fredrick RT, et al. EUS-guided treatment of gastric fundal varices with combined injection of coils and cyanoacrylate glue: a large U.S. experience over 6 years (with video). Gastrointest Endosc 2016;83:1164-1172.

19. Mishra SR, Sharma BC, Kumar A, Sarin SK. Primary prophylaxis of gastric variceal bleeding comparing cyanoacrylate injection and beta-blockers: a randomized controlled trial. J Hepatol 2011;54:1161-1167.
20. Tripathi D, Stanley AJ, Hayes PC, et al. U.K. guidelines on the management of variceal haemorrhage in cirrhotic patients. Gut 2015;64:16801704.

21. Garcia-Tsao G, Abraldes JG, Berzigotti A, Bosch J. Portal hypertensive bleeding in cirrhosis: risk stratification, diagnosis, and management: 2016 practice guidance by the American association for the study of liver diseases. Hepatology 2017;65:310-335.

22. So H, Park do H, Jung K, Ko HK. Successful endoscopic ultrasound-guided coil embolization for severe duodenal bleeding. Am J Gastroenterol 2016;111:925.

23. Sharma M, Somasundaram A. Massive lower GI bleed from an endoscopically inevident rectal varices: diagnosis and management by EUS (with videos). Gastrointest Endosc 2010;72:1106-1108.

24. Weilert F, Shah JN, Marson FP, Binmoeller KF. EUS-guided coil and glue for bleeding rectal varix. Gastrointest Endosc 2012;76:915-916.

25. Tsynman DN, DeCross AJ, Maliakkal B, Ciufo N, Ullah A, Kaul V. Novel use of EUS to successfully treat bleeding parastomal varices with N-butyl-2-cyanoacrylate. Gastrointest Endosc 2014;79:1007-1008; discussion 1008 .

26. Buscaglia JM, Dray X, Shin EJ, et al. A new alternative for a transjugular intrahepatic portosystemic shunt: EUS-guided creation of an intrahepatic portosystemic shunt (with video). Gastrointest Endosc 2009;69:941-947.

27. Schulman AR, Ryou M, Aihara H, et al. EUS-guided intrahepatic portosystemic shunt with direct portal pressure measurements: a novel alternative to transjugular intrahepatic portosystemic shunting. Gastrointest Endosc 2017;85:243-247.

28. Park TY, Seo DW, Kang HJ, et al. Endoscopic ultrasonography-guided placement of a transhepatic portal vein stent in a live porcine model. Endosc Ultrasound 2016;5:315-319.

29. Levy MJ, Wong Kee Song LM, Farnell MB, Misra S, Sarr MG, Gostout CJ. Endoscopic ultrasound (EUS)-guided angiotherapy of refractory gastrointestinal bleeding. Am J Gastroenterol 2008;103:352-359.

30. Gonzalez JM, Ezzedine S, Vitton V, Grimaud JC, Barthet M. Endoscopic ultrasound treatment of vascular complications in acute pancreatitis. Endoscopy 2009;41:721-724.

31. Gonzalez JM, Giacino C, Pioche M, et al. Endoscopic ultrasound-guided vascular therapy: is it safe and effective? Endoscopy 2012;44:539-542.

32. Gamanagatti S, Thingujam U, Garg P, Nongthombam S, Dash NR. Endoscopic ultrasound guided thrombin injection of angiographically occult pancreatitis associated visceral artery pseudoaneurysms: case series. World J Gastrointest Endosc 2015;7:1107-1113.

33. Law R, Fujii-Lau L, Wong Kee Song LM, et al. Efficacy of endoscopic ultrasound-guided hemostatic interventions for resistant nonvariceal bleeding. Clin Gastroenterol Hepatol 2015;13:808-812.e1. 